\title{
Digitale Büroarbeit - Mit Cloud-Lösungen durch die Corona-Krise
}

\author{
Jens Büscher $\cdot$ Jana Treptow 1 D AMAGNO
}

Eingegangen: 10. September 2020 / Angenommen: 25. September 2020 / Online publiziert: 16. Oktober 2020

(C) Springer Fachmedien Wiesbaden GmbH, ein Teil von Springer Nature 2020

Zusammenfassung Die digitale Transformation stellt unsere Gesellschaft vor einen bedeutenden Paradigmenwechsel, hat unsere Arbeitswelt bereits tiefgreifend verändert und wird dies auch in Zukunft fortführen. Die Corona-Pandemie hat diesen Digitalisierungsschub in den vergangenen Monaten enorm begünstigt. Viele Unternehmen mussten ins Home-Office umziehen, um die Gesundheit ihrer Mitarbeiter zu schützen und ihre Existenz zu sichern. Da von zu Hause aus der Zugriff auf die lokalen Akten im Büro kaum möglich ist, sind viele Unternehmen in den vergangenen Monaten verstärkt dazu übergegangen, ihre Daten und Dokumente zu digitalisieren, sie sicher in der Cloud zu speichern und sie unter anderem mittels innovativer Enterprise Content Management-Lösungen immer und von überall abrufen und bearbeiten zu können. Drei von vier Unternehmen setzen nach Angaben des Branchenverbandes Bitkom bereits auf Cloud Computing und die Tendenz ist steigend. In Zukunft soll die verstärkte Konzentration auf die Multi-Cloud-Strategie Unternehmen bei der Verfolgung ihrer Ziele unterstützen und zu einer maximalen Wertschöpfung beitragen.

Schlüsselwörter Digitalisierung $\cdot$ Cloud Computing $\cdot$ Corona-Krise $\cdot$ HomeOffice $\cdot$ Enterprise Content Management

J. Büscher $\cdot$ J. Treptow $(\bowtie)$

Oldenburg, Deutschland

E-Mail: jtr@amagno.de

J. Büscher

E-Mail: jbu@amagno.de 


\section{Digital Office Work-Through the Corona Crisis with Cloud Solutions}

Abstract The digital transformation presents our society with a significant paradigm shift, has already profoundly changed our working world and will continue to do so in the future. The corona pandemic has been an enormous boost to this digitalization drive in recent months. Many companies have had to move to home offices in order to protect the health of their employees and secure their livelihood. Since it is virtually impossible to access local files in the office from home, many companies have increasingly switched to digitizing their data and documents, storing them securely in the cloud and being able to access and edit them anytime, anywhere, using innovative enterprise content management solutions. According to the industry association Bitkom, three out of four companies already rely on cloud computing and the trend is rising. In the future, the increased focus on the multi-cloud strategy should help companies to pursue their goals and contribute to maximum value creation.

Keywords Digitalization · Cloud Computing · Corona crisis · Home Office · Enterprise Content Management

\section{Corona hat die Sichtweisen auf die Digitalisierung verändert}

Die Corona-Krise hat den Stellenwert der Digitalisierung weiter vorangetrieben. Anstelle von physischen Meetings werden die Mitarbeitenden nun mit Tools betraut gemacht, die virtuelle Konferenzen und das Arbeiten von zu Hause aus ermöglichen (Borgmann 2020, S. 21). Bitkom-Präsident Achim Berg sieht das Aufbrechen der bestehenden Strukturen als dringende Notwendigkeit an:

Die Corona-Pandemie und die drastischen Beeinträchtigungen des öffentlichen Lebens erzwingen ein radikales Umdenken in der Kultur vieler Unternehmen. Noch stärker gefordert sind öffentliche Arbeitgeber, für die Homeoffice oft ein Fremdwort ist. Digitale Technologien sind der Schlüssel, um die Arbeitsfähigkeit von Wirtschaft und öffentlichen Einrichtungen wie Ämtern und Schulen auch in dieser außerordentlichen Krisensituation zu gewährleisten. (Bitkom 2020a)

Besonders deutlich wird der von Berg beschriebene Paradigmenwechsel bei der Betrachtung der Nutzungshäufigkeit von Clouddiensten. Laut einer repräsentativen Umfrage des Branchenverbandes Bitkom Research im Auftrag der KPMG AG, nutzten $76 \%$ der befragten Unternehmen im vergangenen Jahr Rechenleistungen aus der Cloud. 2018 waren es 73 und 2017 erst 66\%. Ein stetiger Anstieg zu Cloudlösungen ist also deutlich erkennbar (Pols und Heidkamp 2020, S. 5).

Auch für die Cloudanbieter sind die Auswirkungen der Corona-Krise deutlich spürbar. Als Anbieter für digitales Dokumentenmanagement und Enterprise Content Management merkt auch AMAGNO, dass sich die Sichtweise der Menschen allmählich verändert: „Auch wir als Cloudanbieter merkten insbesondere durch die schlagartig rapide ansteigende Menge an Interessenten, dass sich ein Paradigmen- 
wechsel auf den Weg macht.“ (Jens Büscher, persönliche Kommunikation, 20. Juli 2020).

Die Covid-19-Pandemie lässt sich sehr gut mit einem Tsunami vergleichen. Die rasant ansteigenden Fallzahlen, der unmittelbare Lockdown und die damit verbundenen finanziellen Konsequenzen haben unserer Gesellschaft und ihrer Wirtschaft in den vergangenen Monaten einiges abverlangt und von heute auf morgen unseren Alltag und unsere Routinen tiefgreifend verändert (Handelsblatt 2020). Arbeitsabläufe mussten grundlegend neugestaltet und zusätzlich an den aktuellen Gesundheits- und Arbeitsschutz angepasst werden. Darüber hinaus blieb der ungewisse Blick in die Zukunft. Bundeswirtschaftsminister Peter Altmaier sieht in dem Digitalisierungsschub eine gute Möglichkeit, um die Konjunktur aus ihrem Winterschlaf zu wecken und das Land so substanziell und langfristig voranzubringen:

Neben einem Konjunkturprogramm müssen wir auch strukturell die Wettbewerbsfähigkeit der deutschen Wirtschaft verbessern. Dazu gehören Verbesserungen der IT-Infrastrukturen, Beschleunigung von Planungsverfahren und eine viel stärker digitalisierte Verwaltung. Wir müssen jetzt den durch die Krise ausgelösten Digitalisierungsschub nutzen, um die Digitalisierung konsequent weiter zu forcieren. Unser Ziel muss es sein, hier gegenüber anderen Ländern aufzuholen, damit Unternehmen künftig sicher auf Clouds zurückgreifen können, unsere Kinder in jeder Schulklasse qualitative hochwertige digitale Bildungsangebote in Anspruch nehmen können und Verwaltungsdienstleistungen digital zur Verfügung stehen. (Altmeier 2020)

Während des Lockdowns waren es die digitalen Technologien, die das öffentliche Leben am Laufen hielten - ob im Home-Office, beim Home-Schooling oder OnlineShopping (Bitkom 2020d, S. 3). Unternehmen, die zu Beginn der Krise hauptsächlich noch papierbasiert arbeiteten, traf der Schlag besonders stark, da sie durch den Lockdown und den Rückzug ins Home-Office nahezu handlungsunfähig wurden. Neben der schwachen Leistungsfähigkeit der digitalen Infrastruktur mancherorts und dem unterschiedlich ausgeprägtem Digitalisierungsgrad der Unternehmen, lagern viele Unternehmen ihre wichtigen Dokumente noch in physikalischer Form in den Schränken, sodass sie diese nicht auf digitalem Wege abrufen konnten (Kratz 2020). Diese Tatsache führte bei einer Vielzahl von Entscheiderinnen und Entscheidern zu einer Neujustierung ihrer bisherigen Einstellung zur Digitalisierung. Der zunehmende Digitalisierungsdruck auf Wirtschaft und öffentliche Hand durch Corona war auch ein Aufbruchssignal für das digitale Zeitalter, von der die Unternehmen langfristig profitieren können (Kohlmann 2020).

„Wir selbst waren in der Phase des Lockdowns sehr gut aufgestellt, da wir seit zwei Jahren massiv unser Geschäftsmodell auf monatliche Abo-Basis mit damit verbundenen monatlichen soliden Umsätzen umstellen und damit die wirtschaftlichen Folgen überschaubar blieben“, fasst AMAGNOs Geschäftsführer Jens Büscher (persönliche Kommunikation, 20. Juli 2020) zusammen. Trotz dessen blieb das Unternehmen von den Auswirkungen der Corona-Krise nicht verschont. Büscher sorgte sich um die Gesundheit seiner Mitarbeiterinnen und Mitarbeiter, aber auch um die Arbeitsfähigkeit des Unternehmens. Um das Unternehmen zu schützen, entschied sich AMAGNOs Geschäftsführer schlussendlich, alle Mitarbeitende bis auf Weiteres 
ins Home-Office zu schicken (Jens Büscher, persönliche Kommunikation, 20. Juli 2020).

\section{Digitalisierte Prozesse als Lösung in der Krise}

Während analog arbeitende Unternehmen im Zuge der Krise ihre Prozesse vollständig überarbeiten und ihre Strukturen so verändern mussten, um von zu Hause aus arbeiten zu können, stellte Digitalunternehmen der Umzug ins Home-Office vor keine besonders große Hürde, da viele bereits mit cloudbasierten Anwendungen arbeiteten und so ihre Arbeit zeit- und ortsungebunden flexibel erledigen konnten (Teniz 2020). Je nach Anbieter ist die Inbetriebnahme einer solchen Lösung innerhalb von wenigen Stunden bis hin zu einigen Wochen möglich. Das ist abhängig von der jeweiligen Betriebsart der Anwendung. Wird die Installation der Software innerhalb eines Unternehmens als On-Premise-Modell auf den Servern der eigenen Rechenzentren installiert und mit den eigenen Ressourcen des Unternehmens, wie etwa Hard- und Software, Personal und Wartung, selbst betrieben, ist der Aufwand für die Cloudvariante hingegen deutlich geringer. Werden die Daten mittels externer ITInfrastruktur bei einem Cloudanbieter gelagert, entfallen unter anderem die Kosten für Anschaffung und Bereitstellung von benötigter Hard- und Software, Ausgaben für das Personal und anfallende Betriebskosten. Hinzu kommt, dass den Nutzern in einer Public Cloud die IT-Services über das Internet zur Verfügung stehen und sie keinen direkten Zugriff auf die IT-Infrastruktur des Unternehmens benötigen. Bei On Premise-Varianten einer Anwendung ist das genaue Gegenteil der Fall (Ziebell et al. 2018, S. 128; Von Faber und Behnsen 2018, S. 52).

Welche Lösung am geeignetsten ist, hängt von den eigenen Ressourcen und den eigenen Zielen ab und kann nicht pauschal beantwortet werden. On Premise bietet sich zum Beispiel an, wenn hochsensible Daten auf den Anwendungen gespeichert werden, da Kontrolle bei dieser Variante vollständig im Unternehmen verbleibt, während die Anwenderorganisationen mit der Cloud-Variante wesentlich mehr Flexibilität haben und ihre IT-Kosten für Hard- und Software, Personal und Wartung reduzieren (Von Faber und Behnsen 2018, S. $53 \mathrm{f}$.).

Das Sicherheitsrisiko beider Varianten, ist bei beiden Modellen unterschiedlich zu bewerten. Wird die unternehmensrelevanten Informationen bei der On-PremiseVariante zwar auf den eigenen Servern gespeichert, hat die Anwenderorganisation oftmals jedoch gar nicht die Ressourcen, alle sicherheitsspezifischen Zertifikate zu erwerben, regelmäßig nach Updates zu suchen oder Wartungen durchzuführen (ebd., S. 51). Das ist nur einer von vielen Gründen, weshalb sich viele Anwender für den Wechsel ihrer Daten in die Cloud entscheiden. Hinsichtlich der allgemeinen Sicherheitsaspekte einer Cloud-Lösung wird deutlich, dass die Sicherheitsvorfälle zwar abnehmen, aber die Verdachtsfälle steigen. Lediglich jedes vierte Unternehmen registrierte im vergangenen Jahr überhaupt keine Vor- oder Verdachtsfälle in der Cloud. Nur zwei von fünf Public-Cloud-Nutzer konnten auf ein Jahr ganz ohne Sicherheitsvorfälle zurückblicken. Unternehmen sollten also ihre Sicherheitsprüfungen beschleunigen und ihre Sicherheitsstrategien stetig ausbauen, um mit der Geschwindigkeit der Angreifer mithalten zu können (Pols und Heidkamp 2020, S. 25). Dieser 
Trend ist auch bei vielen Cloud-Nutzern erkennbar. Rund $77 \%$ der Unternehmen in der Cloud besitzen ein cloudspezifisches Sicherheitskonzept und passen es regelmäBig an, um vor etwaigen Angriffen gewappnet zu sein. Im vergangenen Jahr lang diese Zahl erst bei 39\% (ebd., S. 26).

Der Sicherheitsaspekt in der Cloud ist jedoch noch umstritten. Während die einen salopp sagen, dass eine hundertprozentige Sicherheit niemals vorherrschen kann, sagen die anderen, dass Sicherheit ein Prozess ist, bei dem funktionierende und nachhaltige Prozesse den Sicherheitszustand erhöhen und es ein sich stetig weiterentwickelter Prozess ist, der mit den ersten IT-Sicherheitsgesetzen bereits Form annimmt. Tatsache ist, dass Anbieter digitaler Dienste nach §8c BSIG zur Einhaltung von IT-Sicherheitsstandards und -maßnahmen verpflichtet sind und erhebliche Sicherheitsvorfälle gem. §8c III BSIG an das BSI zu melden (Adelmeyer et al. 2018, S. 9 f.). Werden die geschäftskritischen Daten eines Unternehmens bei einem Provider gespeichert, dessen Rechenzentren in Deutschland oder im europäischen Wirtschaftsraum stehen, greift das deutsche Recht bzw. EU-Recht und die damit verbundenen Regularien. Im Vergleich zu den USA, hat Deutschland nicht zuletzt aufgrund der DSGVO, strenge Auflagen zu erfüllen, was den Schutz von persönlichen Daten betrifft. Das Bundesdatenschutzgesetz ist eines der strengsten Gesetze zur informationellen Selbstbestimmung und die Nutzung personenbezogener Daten ist hierzulande strengstens reguliert und bedarf der ausdrücklichen Erlaubnis der betroffenen Person. Im angloamerikanischen Raum ist dies genau andersherum. Zur Terrorbekämpfung verschaffen sich die US-Geheimdienste gemeinsam mit der USamerikanischen Gesetzgebung dort so einen Zugang zu personenbezogenen Daten. Wer seine Daten also auf deutschen Servern speichert, ist buchstäblich auf der sicheren Seite (Abolhassan 2016, S. 22). Da Sicherheit einer der Schlüsselfaktoren ist, der über die Akzeptanz von Cloud-Diensten entscheidet, liegt der Fokus der Cloudanbieter auf der der Sicherheit personenbezogener Daten. So besitzen viele Cloudanbieter zahllose Zertifikate, um die Sicherheitsrichtlinien zu erfüllen und ihre Kunden nicht zu verlieren. Damit die Cybersicherheit gewährleistet werden kann, arbeiten besonders die großen Anbieter, wie beispielsweise Microsoft, rund um die Uhr daran, etwaige Bedrohungen zu identifizieren und schnell darauf zu reagieren, um die großen Fauxpas zu vermeiden (Microsoft 2020).

Ein weiterer entscheidender Faktor ist die Tatsache, dass die Cloud die Basis für digitalisierte Geschäftsmodelle und -prozesse ist, die Unternehmen in Zukunft prägen wird, da unsere Welt mehr und mehr an Konnektivität gewinnt (Abolhassan 2016, S. 15). Deshalb steigen nicht nur die Zahlen von Cloud-Computing-Services seit einigen Jahren drastisch, sondern auch die Umsatzprogosen (Gartner 2020, S. 3).

Besonders während der Corona-Krise war ein deutlicher Anstieg von Cloud-Lösungen erkennbar. Einer der Hauptargumente, die für die Cloud sprechen, ist neben den erheblichen Kosteneinsparungen die flexible orts- und zeitunabhängige Zugriffsmöglichkeit auf die gewünschten Dokumente. Mit einer stabilen Internetverbindung lassen sich alle benötigten Informationen unmittelbar abrufen - ob im Office, von zu Hause aus oder während einer Dienstreise. Darüber hinaus bieten Geschäftsprozesse in der Cloud für Unternehmen die Möglichkeit, Marktanteile hinzuzugewinnen und nachhaltig erfolgreich zu sein (ERP Management 2020). 
Das Cloud-Computing ist nicht nur in den vergangenen Jahren für viele Unternehmen zu einem wichtigen Bestandteil ihrer IT-Infrastruktur geworden, sondern außerdem zu einem bedeutenden Treiber der Digitalisierung (Pols und Heidkamp 2020, S. 3). Allein zwischen 2016 und 2018 stieg die Zahl der Cloud-Anwender von 65 auf $73 \%$ und der Anteil derjenigen Unternehmen, die das Cloudmodell ablehnten, sank von 17 auf $8 \%$ (ERP Management 2020). Hinzu kommt, dass Unternehmen die Cloud auch zunehmend für unternehmenskritische Daten nutzen.

Klein- und mittelständische Unternehmen stellen sich nicht mehr die Frage, ob sie in die Cloud gehen wollen, sondern nur noch wie, denn eine einfache Installation, wie die Installation eines Kaffeevollautomaten ist es allerdings nicht (ebd. 2020, S. 18 ff.). Der Weg in die Cloud kann nicht von heute auf morgen bestritten werden, sondern muss gut durchdacht und geplant werden, um einen langfristigen Unternehmenserfolg zu erzielen. Auch zeigte sich bei der Erhebung, dass sich die IT-Budgets für die Aufwendungen von Cloud-Computing steigen. Allein 2019 wendeten die Unternehmen rund $20 \%$ ihres gesamten IT-Budgets für Public-CloudLösungen auf. Im Vergleich zum Vorjahr ist das ein Anstieg um zwei Prozentpunkte. Und die Tendenz ist steigend (ebd. 2020, S. 13). Die Public-Cloud-Nutzer berichten zudem, dass sich der Cloud-Einsatz positiv auf ihr IT-Budget auswirkt, da neben den allgemeinen Vorteilen die IT-Kosten durch den Einsatz einer Cloud um knapp $24 \%$ gesunken sind (Pols und Heidkamp 2020, S. 3).

\section{Cloudbasierte Enterprise Content Management-Lösungen als Zukunftstechnologie}

In den Unternehmen besteht unweigerlich einer Vielzahl von Datenablagen für Dokumente. Ob auf herkömmlichem Papier, in Fachanwendungen, E-Mails oder auf Netzlaufwerken und lokalen Datenträgern: je höher die Anzahl an Datenablagen, desto fehleranfälliger ist auch die Informationsbeschaffung und -versorgung. Hinzu kommt, dass Medienbrüche Verzögerungen und abkömmlichen Ressourcenaufwand in der Bearbeitung der Vorgänge mit sich bringen und es aufgrund der hybriden Datenerhaltung nicht selten zu Informationslücken kommt und hohe Datenredundanzen entstehen (Bosse und Zink 2019, S. 241). Ganz zu schweigen von den widerrechtlichen Änderungen oder Löschungen von Belegen und Dokumenten (Verband elektronische Rechnung 2020). Das ist für alle Beteiligten nicht nur auf zeitlicher Ebene ein Ärgernis, sondern überdies auch noch äußerst ineffizient, demotivierend, kostenintensiv und teilweise mit Compliance-Problemen und Rechtsverstößen verbunden. Wenn rund 450 Mitarbeitende jeden Tag 15 min für die Suche nach Dokumenten aufwenden, führt das bei einem Durchschnittslohn von $25 €$ pro Stunde, zu einem Jahresverlust von mehr als 700.000€ (Konica Minolta Österreich 2018).

Viele Mitarbeiterinnen und Mitarbeiter sind so an ihre analogen Strukturen und Prozesse gewöhnt, dass sie sich nicht nach effizienteren Arbeitsweisen umsehen. Hinzu kommt die Angst, die sie hemmt, ihre historischen Arbeitsweisen zu verlassen und sich digitalen Anwendungen zuzuwenden, da sie fürchten, ihren Arbeitsplatz zu verlieren. Diese Ängste sind jedoch keineswegs berechtigt, da Menschen auch in Zukunft als Planer und Entscheidungsträger benötigt werden und für die Qualität 
und Sicherheit der Prozesse verantwortlich sind. Zumal diejenigen Länder mit dem höchsten Digitalisierungs- und Automatisierungsgrad die niedrigsten Arbeitslosenquoten aufweisen (Larsen 2019, S. 315 f.). Digitale und (teil-)automatisierte Systeme sollen uns lediglich bei unserer täglichen Arbeit unterstützend zur Seite stehen und uns den Raum schaffen, um unsere tatsächlichen Tätigkeiten auszuführen, uns auszutauschen, effizient zu arbeiten und schneller an die gewünschten Informationen zu gelangen (De Brabandt 2019, S. 45).

Die historische Arbeitsweise wird zum Beispiel dann problematisch, wenn eine digitale Rechnung analog archiviert wird. Denn der Gesetzgeber sieht vor, dass Rechnungen zwingend in dem Format archiviert werden, in dem sie auch im Unternehmen eingehen (Verband elektronische Rechnung 2020). Um dem entgegenzuwirken und eine zentrale Bereitstellung aller unternehmensrelevanter Dokumente unter Einhaltung gültiger Rechtsnormen zu ermöglichen, stellt der Anbietermarkt sogenannte Enterprise Content Management-Lösungen (ECM) zur Verfügung. Durch innovative Techniken und Prozesse bieten sie die Möglichkeit, unternehmensrelevante Daten in Form von nicht, schwach oder strukturierter Dokumente aus verschiedenen Quellen zusammenzutragen. Mithilfe einer Rollenvergabe werden sie so unmittelbar den entsprechenden Mitarbeiterinnen und Mitarbeitern zugeordnet (Riggert 2019, S. 3).

Als Teilbereich der ECM-Lösungen arbeiten Unternehmen oftmals mit innovativer Dokumentenmanagement Software (DMS), die sich auf die Verwaltung von Dokumenten konzentriert. Was als reine Dokumentenverwaltung begann, weitete sich im Laufe der Jahre immer weiter aus. So stehen bei den heutigen Dokumentenmanagementsystemen vor allem die Abbildung von und Arbeit mit Workflows, elektronischen Signaturen, digitalen Akten, aber auch das Management von Wissen im Vordergrund (ebd., S. 65). Der Branchenverband Bitkom (2016a) ordnet das Dokumentenmanagement als System ein, dass ,,[... zur Organisation und Koordination der Entwicklung, Verarbeitung, Überwachung und Verteilung von Dokumenten aller Art über ihren gesamten Lebenszyklus von ihrer Entstehung bis zu ihrer Vernichtung [dient]“" (S. 8).

Beide Systeme haben gemein, dass sie alle Dokumentenströme erfassen, sie verwalten und sie durchsuchbar machen. Außerdem regeln sie die Geschäftsprozesse und sorgen für die notwendige Langzeitarchivierung.

\section{ECM schafft effizientere Arbeitsabläufe}

Wie sehr Ausnahmesituationen wie der Corona-Lockdown die Geschäftsprozesse eines Unternehmens beeinträchtigen können, hängt von dessen Digitalisierungsgrad ab. Besonders erfolgskritisch ist der Post- und Rechnungseingang, da viele Unternehmen von der örtlichen Anwesenheit ihres Personals für den analogen Posteingang angewiesen sind. Schlussendlich liegt die Verantwortlichkeit bei ihnen, die Briefpost zu öffnen, Rechnungen, Aufträge, Angebote und die allgemeine Post zu selektieren, zu sortieren und an die entsprechenden Stellen weiterzuleiten. Geschieht das nicht, steht der Erfolg des Unternehmens auf dem Spiel. Die Corona-Krise machte deutlich, dass der Umzug ins Home-Office gut überlegt und wohl strukturiert sein muss, damit die Vielzahl an Existenzen nicht gefährdet werden. Erfolgt die Bearbeitung der Ein- 
gangspost auf digitalem Weg und ist die Rechnungsbearbeitung (teil-)automatisiert sichergestellt, entfallen ungewünschte Mahnungen oder entgangene Skontofristen (Stadler 2020).

Insbesondere die Verarbeitung von Rechnungen hat in den letzten Jahren einen gewaltigen Digitalisierungsschub erhalten. Diesen Aufwärtstrend der elektronischen Rechnungsbearbeitung haben nicht zuletzt Gesetzesänderungen geprägt, die zu massiven Vereinfachungen in der Bearbeitung von digitalen Rechnungen geführt haben. Auch die zahlreichen Scandienstleister, die die Digitalisierung und Verwaltung des Posteingangs eines Unternehmens übernehmen und die Dokumente digital und ortsunabhängig mithilfe spezifischer Schnittstellen dem Unternehmen zur Verfügung stellen, haben die Entwicklung weiter vorangetrieben. So lohnt sich die Digitalisierung der Eingangspost für Unternehmen nicht nur auf monetärer und zeitlicher Ebene, sondern außerdem aus Sicht des ortsunabhängigen Zugriffs. Denn wer zeitund ortsungebunden auf Dokumente zugreifen kann, kann schnelle Antworten geben und ermöglicht effizienteres Arbeiten, da das gesamte Wissen zentral in dem Enterprise Content Management gespeichert wird (Bitkom 2016b, S. 4).

Nicht selten verfügen Unternehmen über Unmengen an Prozesse und Workflows, um ihre Produkte zu fertigen oder Dienstleistungen zu erbringen. Durch die Flexibilität und Vielfalt heutiger ECM-Lösungen, ist auch die Abbildung komplexer Workflows problemlos umsetzbar. Bereits fertige Abläufe für klassische Szenarien stehen in den Bereichen Vertrags- und Rechnungswesen, Personal- und Bewerbermanagement oder aber E-Mail-Archivierung zur Verfügung und können je nach Wunsch an die individuellen Bedürfnisse des Unternehmens angepasst werden. Die Prozesse lassen sich anschließend mit einem Cloud-basierten Enterprise Content Management an jedem Ort, über jedes Gerät und zu jeder Zeit starten, bearbeiten und überblicken. Durch diese Transparenz und Nachvollziehbarkeit können unmittelbare Auskünfte erteilt und etwaige Eskalationen oder bevorstehende Fristen eingesehen werden, ehe der Grenzpunkt überschritten ist. So reduzieren sich Laufwege und Liegezeiten auf ein Minimum und die Kunden- und Servicequalität des Unternehmens steigt. Durch die vordefinierten digitalen Workflows erreicht ein Unternehmen zudem hohe Qualitätsstandards, die sich automatisch auch in einem guten Qualitätsmanagement widerspiegeln (QZ 2020).

Nebst diesen Vorteilen stellt die Cloud-Software Daten in Echtzeit bereit und ist deshalb für eine gemeinsame Kollaboration an unterschiedlichen Standorten interessant. Durch die individuelle Skalierbarkeit der Cloud-Variante einer Software, können Anwenderinnen und Anwender nach Bedarf hinzugefügt werden oder modular konzipierte Anwendung ohne Weiteres erweitert werden. Insbesondere für kleine und mittelständische Unternehmen ist die Cloud-Variante eine gute Option, da sie mit dem Unternehmen wachsen kann und so Investitionskosten für Software und Server entfallen bzw. besser skaliert werden können (ERP Management 2019).

\section{Der Tag nach dem Corona-Tsunami - eine Zukunftsprognose}

Die Corona-Krise hat in den vergangenen Monaten so manches Unternehmen vor eine echte Zerreißprobe gestellt. Prozesse mussten schnell umgestellt und neu ge- 
dacht werden. Die Umstrukturierung und Digitalisierung eines Unternehmens und seiner Workflows, die sonst Monate dauerten, mussten binnen weniger Wochen realisiert werden. Die Menschen mussten ihre Existenzängste überwinden und wurden zum Handeln gezwungen. Die angeordneten Maßnahmen der Bundesregierung erforderten die Suche nach geeigneten Tools, die mobiles und flexibles Arbeiten ermöglichen, um die Verbreitung des Virus weiter einzudämmen. Nach Angaben einer Bitkom-Befragung blieb zu Beginn der Corona-Krise Mitte März jeder Zweite ganz oder zumindest teilweise im Home-Office (Treptow 2020; Bitkom 2020a). Für zwei Drittel der Start-Ups in Deutschland hat sich jedoch ihre wirtschaftliche Lage seit Beginn der Corona-Pandemie erheblich verschlechtert. Allein jeder Zweite gibt an, dass die Corona-Krise seine Existenz bedroht (Bitkom 2020b).

Doch viele Unternehmen gehen in Hinblick auf die Bedeutung der Digitalisierung und der Cloud aus dieser Krise auch gestärkt hervor und stehen der Digitalisierung offener gegenüber (Bitkom 2020c). Das Schweizer Unternehmen re:cap beispielsweise. Die re:cap global investors ag ist ein international tätiger unabhängiger M\&ABerater und Asset Manager für erneuerbare Energien: Seit vielen Jahren arbeitet das Dienstleistungsunternehmen bereits mit der Cloud des digitalen Dokumentenmanagements Amagno.

Mit „unserer“ Amagno-Cloud können wir gemeinsam mit unseren Dienstleistern in ganz Europa standortunabhängig und mit sicheren Zugängen in einem System den Betrieb unserer Solar- und Windparks gewährleisten. Durch den Corona-bedingten Wechsel ins Home-Office ergab sich weder für uns noch für unsere Dienstleister unangenehme Veränderungen in den Arbeitsabläufen oder der Bearbeitungszeit. Wir sehen heute noch dringender die Erfordernisse, sich gut mit digitalen Signaturen aufzustellen. Sobald das gelingt, können wir in Zukunft zudem noch unabhängiger und papierloser arbeiten. (Anja Spannaus, persönliche Kommunikation, 24. Juli 2020)

Aber auch der Geschäftsführer Jürgen Hermann der QSC AG, die sich auf die flexible und effiziente Weiterentwicklung von Geschäftsprozessen und -modellen ihrer Kunden spezialisiert hat, sieht in seinem Unternehmen positive Veränderungen durch die Covid-19-Krise:

Wir gehen [...] davon aus, dass in Zukunft mehr Mitarbeiterinnen und Mitarbeiter zumindest situativ, aber sicher auch regelmäßiger als zuvor im HomeOffice arbeiten werden und dass auch der Kundenkontakt öfter und spontaner per Videoconferencing stattfinden wird, so dass Dienstreisen deutlich weniger werden. (Bitkom 2020d, S. 23)

„Wer jetzt als Entscheider nicht sieht, wie relevant die Akzeptanz und Umsetzung einer Digitalisierungsstrategie ist, der hat verloren. Wir müssen lernen, heute die Werkzeuge von Morgen einzusetzen, und nicht morgen die Werkzeuge von gestern“ beschreibt Jens Büscher (persönliche Kommunikation, 20. Juli 2020) die aktuelle Situation. Die Krise hat die Schwachstellen unseres Gesellschaftssystems nochmals deutlich zu Tage getragen und nun geht es darum, Geschäftsprozesse zukünftig flexibler und effizienter zu gestalten, die Präsenzkultur zu überdenken, bisherige Arbeits- und Reisemodelle anzupassen und durch die richtigen regula- 
torischen Schlüsse der Digitalisierung einen weiteren Schub zu verleihen (Bitkom 2020e).

\section{Cloud Computing zukunftsorientiert gedacht}

Die nächste Stufe des Cloud Computing wird in nächster Zeit mit der Multi-CloudNutzung erklommen. Jedes dritte Unternehmen fährt nach Angaben des Bitkom bereits eine Multi-Cloud-Strategie; die Gründe dafür sind mannigfaltig. Zum einen geht es Entscheidern und Nutzen besonders um die Gewährleistung der Performance und Stabilität ihrer eingesetzten Cloud-Lösung. Durch die Bereitstellung von Ersatzressourcen werden Cloud-Ausfälle vermieden und die Ressourcen bei Auslastungsspitzen besser verteilt. Hinzu kommt der stetig wachsende Wunsch der Unternehmen, spezifische Cloud-Anwendungen von verschiedenen Cloud-Providern einzusetzen. Da sich Sicherheitsvorfälle auch in Zukunft wohl kaum vermeiden lassen, sollten Unternehmen sich auch in der kommenden Zeit mithilfe gut durchdachter Sicherheitskonzepte und ihrer kontinuierlichen Anpassung auf etwaige Bedrohungsszenarien vorbereiten. So können sie sich gezielt für den Ernstfall vorbereiten und professionell darauf reagieren (Pols und Heidkamp 2020, S. 31 f.).

Amagnos Gründer und Geschäftsführer hat für seine Firma mittelfristig einen klaren Fahrplan für das zukünftige Arbeiten in der Cloud:

Die Cloud bedeutet den kompletten Umbau einer serverzentrischen Software auf eine komplett Services-basierte Lösung - beispielsweise auf Container-Basis. Dabei wird die monolithische Software in Einzelteile zerlegt, sodass die Lösung in der Cloud beliebig skaliert werden kann. Sie reagiert damit auf hohes Wachstum, hohe Benutzerzahlen und hohe Datenmengen. Ergänzt wird dies Client-seitig mit dem Wegfall einer nativen Client-Anwendung auf eine moderne App-Basis, die aufjedem Endgerät verwendet werden kann. Diese Ziele umfasst unsere Roadmap in den nächsten zwei Jahren. (Jens Büscher, persönliche Kommunikation, 20. Juli 2020)

Mehr als drei von vier Unternehmen arbeiten bereits mit Anwendungen in der Cloud. Die Zahlen deuten auf ein starkes Wachstumspotenzial in der Cloud-Nutzung hin; die Prioritäten von Cloud-Providern und Cloud-Anwendern werden sich zukünftig jedoch verändern. Lag der Schwerpunkt bisheriger Cloud-Projekte in der Betreuung während der Cloud-Implementierung, geht es in Zukunft vermehrt darum, die Anwender zum nächsten Cloud-Reifegrad zu begleiten. Die Integration einer Multi-Cloud-Strategie in die bestehenden Prozesse und Workflows wäre dabei ein möglicher, wegweisender Ansatz für die gleichzeitige Nutzung und Bündelung verschiedener Cloud-Computing-Dienste. Um dieses bestmöglich unterstützen und gewährleisten zu können, ist insbesondere für die Provider eine Roadmap ihrer Cloud-Anwendung unausweichlich, bei der Berücksichtigung bestehender CloudInfrastrukturen und der Anforderung an Security und Compliance integriert werden. Denn die Integrationsfähigkeit neuer Cloud-Lösungen sowie die Interoperabilität neuer Anwendungen mit der bestehenden Infrastruktur gewinnen zunehmend für die Cloud-Anwender an Wichtigkeit (Pols und Heidkamp 2020, S. 32). 


\section{Literatur}

\section{Verwendete Literatur}

Abolhassan F (2016) Was treibt die Digitalisierung? Warum an der Cloud kein Weg vorbeiführt. Springer Gabler, Wiesbaden

Adelmeyer M, Petrick C, Teuteberg F (2018) IT-Risikomanagement von Cloud-Services in Kritischen Infrastrukturen. Springer Vieweg, Wiesbaden

Altmeier P (2020) Digitalisierungsschub statt analogem Strohfeuer. https://www.bitkom.org/Presse/ Presseinformation/Digitalisierungsschub-statt-analogem-Strohfeuer. Zugegriffen: 27. Juli 2020

Bitkom (2016a) Bitkom Digital Office Index. Bitkom, Berlin

Bitkom (2016b) Outsourcing von dokumentenbezogenen Unternehmensprozessen. Scannen und Verarbeitung von Dokumenten durch einen Dienstleister - Ein Leitfaden für die Unternehmenspraxis. Bitkom, Berlin

Bitkom (2020a) Corona-Pandemie: Arbeit im Homeoffice nimmt deutlich zu. https://www.bitkom.org/ Presse/Presseinformation/Corona-Pandemie-Arbeit-im-Homeoffice-nimmt-deutlich-zu. Zugegriffen: 27. Juli 2020

Bitkom (2020b) Corona-Pandemie: Corona-Krise trifft Startups mit voller Wucht. https://www.bitkom.org/ Presse/Presseinformation/Corona-Krise-trifft-Startups-mit-voller-Wucht. Zugegriffen: 31. Juli 2020

Bitkom (2020c) Digitalisierung findet mehr Zuspruch. https://www.bitkom.org/Presse/Presseinformation/ Digitalisierung-findet-mehr-Zuspruch. Zugegriffen: 31. Juli 2020

Bitkom (2020d) Der IT-Mittelstand in Deutschland. IT-Mittelstandsbericht 2020. Bitkom, Berlin

Bitkom (2020e) Positionspapier: Digitale Arbeitswelt nach der Corona-Krise. https://www.bitkom.org/ sites/default/files/2020-06/20200625_digitale-arbeitswelt-nach-der-corona-krise_final.pdf. Zugegriffen: 30. Juli 2020

Borgmann V (2020) Futurehotel - Das smarte resiliente Hotel. Wie hilft die Digitalisierung den Hotels in Zeiten der Corona-Krise und danach? Fraunhofer Verlag, Stuttgart

Bosse C, Zink K (Hrsg) (2019) Arbeit 4.0 im Mittelstand. Chancen und Herausforderungen des digitalen Wandels für KMU. Springer Gabler, Berlin, Heidelberg

De Brabandt C (2019) Verhandeln für Jedermann. In: Buchenau P (Hrsg) Chefsache Zukunft. Was Führungskräfte von morgen brauchen. Springer Gabler, Wiesbaden, S 23-45

Von Faber E, Behnsen W (2018) Joint Security Management: organisationsübergreifend handeln. Mehr Sicherheit im Zeitalter von Cloud-Computing, IT-Dienstleistungen und industrialisierter IT-Produktion. Springer Vieweg, Wiesbaden

Gartner (2020) Gartners forecasts worldwise public cloud revenue to grow $17 \%$ in 2020. https://www. gartner.com/en/newsroom/press-releases/2019-11-13-gartner-forecasts-worldwide-public-cloudrevenue-to-grow-17-percent-in-2020. Zugegriffen: 28. Juli 2020

Handelsblatt (2020) Richemont sieht sich im Corona-,,Tsunami“ - Umsatz bricht ein. https://www. handelsblatt.com/unternehmen/handel-konsumgueter/luxuskonzern-richemont-sieht-sich-im-coronatsunami-umsatz-bricht-ein/25833458.html?ticket=ST-12947902-UbZt0k4O9QbzlMkpY9CL-ap6. Zugegriffen: 27. Juli 2020

Kohlmann T (2020) Digitaler Schub durch Corona. https://www.dw.com/de/digitaler-schub-durch-corona/ a-53186597. Zugegriffen: 27. Juli 2020

Konica Minolta Österreich (2018) Tool für die unternehmensinterne Suche. it\&t, 11/2018, 15. https://www. wiso-net.de/document/ITT__ffd5408e6c026b2b45dba7414f95537475a431f4. Zugegriffen: 27. Juli 2020

Kratz A (2020) Wie Homeoffice bei schlechtem Internet funktioniert. https://www.stuttgarter-zeitung. de/inhalt.corona-krise-wie-homeoffice-bei-schlechtem-internet-funktioniert.3079ffb0-0dae-494c9a6d-e028a2f9ed4c.html. Zugegriffen: 27. Juli 2020

Larsen B (2019) Generationengerechte Unternehmensführung 5.0: Babyboomer und digitale Eingeborene - Unternehmenskultur mit Konfliktpotenzial. In: Buchenau P (Hrsg) Chefsache Zukunft. Was Führungskräfte von morgen brauchen. Springer Gabler, Wiesbaden, S 313-332

Management ERP (2019) Zukunftstechnologie Cloud Computing. ERP Manag 2/2019:38-40

Management ERP (2020) Digitalisierung des Mittelstands Wertvolle Potenziale mit Cloud Computing heben. ERP Manag 1/2020:52-53

Microsoft (2020) Stärken Sie Ihren Sicherheitsstatus mit Azure. https://azure.microsoft.com/de-de/ overview/security/. Zugegriffen: 29. Juli 2020 
Pols A, Heidkamp P (2020) Cloud-Monitor 2020. Die Integrationsfähigkeit und Interoperabilität der Cloud stärken. KPMG, Bitkom Research, Berlin

QZ (2020) Effizienter mit der Cloud. Quintessenz Zahntech 3/2020:46

Riggert W (2019) ECM - Enterprise Content Management. Konzepte und Techniken rund um Dokumente. Springer Vieweg, Wiesbaden

Stadler A (2020) Post- und Rechnungseingang krisensicher digitalisieren. https://www.ecmguide.de/ input-output/postbearbeitung/post-und-rechnungseingang-krisensicher-digitalisieren-24894.aspx. Zugegriffen: 29. Juli 2020

Teniz M (2020) Homeoffice - Herausforderung für Management und Technik. https://www.it-markt.ch/ news/2020-05-04/homeoffice-herausforderung-fuer-management-und-technik. Zugegriffen: 27. Juli 2020

Treptow J (2020) Mit einer 360-Grad-Rolle vorwärts ins Home-Office. https://amagno.de/mit-einer-360grad-rolle-vorwaerts-ins-home-office/38663/. Zugegriffen: 29. Juli 2020

Verband elektronische Rechnung (VeR) (2020) 12 Regeln für den GoBD-konformen elektronischen Rechnungsaustausch. https://www.verband-e-rechnung.org/archiv/fachartikel/12-regeln-fuer-den-gobdkonformen-elektronischen-rechnungsaustausch/. Zugegriffen: 28. Juli 2020

Ziebell R-C, Schoeneberg K-P, Schultz M, Garrigós JA, Perello-Marin MR (2018) Vom traditionellen Personalmanagement hin zu e-HRM in der Cloud Implementierungsansätze einer digitalen HR-Transformation. In: Reinheimer S (Hrsg) Cloud Computing. Die Infrastruktur der Digitalisierung. Springer Vieweg, Wiesbaden, S 113-139

\section{Weiterführende Literatur}

Bär C, Krug P (2018) Strategische Ausrichtung und Ziele der DATEV eG zur Forcierung von Cloud Computing. In: Reinheimer S (Hrsg) Cloud Computing. Die Infrastruktur der Digitalisierung. Springer Vieweg, Wiesbaden, S 43-53

Neugebauer R (2018) Digitalisierung. Schlüsseltechnologien für Wirtschaft und Gesellschaft. Springer Vieweg, München

Reinnarth J, Schuster C, Möllendorf J, Lutz A (2018) Chefsache Digitalisierung 4.0. Springer Gabler, Wiesbaden

Verband elektronische Rechnung (VeR) (2020) Alles Wissenswerte zum E-Rechnungs-Gesetz. https:// www.verband-e-rechnung.org/e-rechnungsgesetz/. Zugegriffen: 24. Juli 2020 